
HERE TO EDIT) $<$

\title{
A Tightly Integrated Multilayer Battery- Antenna for RFID Epidermal Applications
}

\author{
M. C. Caccami, M. P. Hogan, M. Alfredsson, G. Marrocco and J. C. Batchelor, Senior Member
}

\begin{abstract}
For the acceptance of bio-integrated devices in daily life, radio-systems must be developed that are minimally invasive to the skin, and they must have ultra-low profile local power sources to support data-logging functionality without compromising shape-conformability. This contribution proposes a tightly integrated multilayer battery-antenna system $(65 \times$ $23 \mathrm{~mm}^{2}$ ) that is ultra-thin (just $200 \mu \mathrm{m}$ ), flexible, and lighter than $1 \mathrm{~g}$, making it suitable for epidermal applications. The negative electrode (anode) current collector of the battery is a Radiofrequency Identification (RFID) tag antenna coated by a conductive polymer (Pedot:PSS) working as anode material. Since the battery is a dynamic device, subjected to discharging, the antenna design must include the variable dielectric properties of the conductive polymer that are here first characterized in the UHF band for real charge/discharge battery conditions. The communication performance of the prototype composite device is hence evaluated through the measurement of the realized gain of the tag antenna $(-19.6 \mathrm{dBi}$ at $870 \mathrm{MHz})$ when it is placed directly onto a volunteer's forearm. The read range of $1.3-3 \mathrm{~m}$ is suitable for occasional data download from the epidermal data-logger when the user comes close to a reader-equipped gate.
\end{abstract}

Index Terms-Conductive polymer, Integrated battery-antenna device, Planar battery, RFID technology, Wearable antenna

\section{INTRODUCTION}

$\mathrm{O}$ VER the past decade, significant progress in the emerging field of bio-integrated wearable electronics has resulted in a new class of minimally invasive flexible and stretchable systems suitable to interface with the curved surfaces of the human body [1], [2]. There is also a growing interest in developing new form-factor local energy sources for easy integration with the human body to enable uninterrupted activity of the sensor even in the absence of a remote interrogator [3], [4], thus working as a data-logger. Indeed, wearable and epidermal devices must meet the increasing demands of detecting multiple parameters simultaneously, while supporting data transmission over several meters in order to provide reliable in-room tracking [5].

Conventional power sources, such as lithium ( $\mathrm{Li}$ ) ion and alkaline batteries, offer high energy densities, long cycle life,

Paper submitted on 16 August 2017, reviewed on 17 October 2017 and 9 November 2017, and resubmitted on 22 November 2017; accepted on 27 November 2017.

M. C. Caccami and G. Marrocco are with the Pervasive Elecromagnetics Lab, www.pervasive.ing.uniroma2.it, University of Roma Tor Vergata, Italy. email: good shelf life, rapid charge and discharge capability, and high energy efficiency. Nevertheless, this family of battery is bulky and rigid and, thus, cannot meet some of the main wearable sensor flexibility and compactness requirements for on-body use. Moreover, the metallic parts of these batteries, generally, cause mismatch of the tag antenna impedance with a resulting decrease in the read distance of the wearable device.

Advancements in materials science and digital printing techniques have helped address the intrinsic physical mismatch between widely used rigid devices and the soft and curvilinear surface of human skin, thereby enabling unprecedented options to achieve low-profile batteries that are biocompatible and can be eco-friendly. Such batteries require no metallic casing [6], can include electrode materials printed or coated onto flexible substrates [7]. They can also comprise organic materials [8] and, in particular, conductive polymers with reasonable electrical conductivity to preserve the antenna performance, good ionic conductivity, and ease of manufacture [9]. However, it is still extremely challenging to design and fabricate a device capable of integrating an antenna and a battery that, besides providing the required capacity and discharge rate for wearable and wireless electronics, is also sufficiently mechanically flexible [10], [11]. This goal can only be met by a physical-level antenna design method accounting for the intimate interaction with the battery time-variant materials.

Following the recent proof of concept simulations in [12] and [13], concerning a first attempt to achieve a composite batteryantenna structure for short-range communications, this contribution now introduces the design and test of the first working example of a battery embedded into the antenna substrate, where a Radio Frequency Identification (RFID) tag is coated by a conductive polymer (Pedot:PSS) so that it may work as the anode of the battery. While Pedot:PSS has already been proposed for antenna fabrication, here it is doped with a variable amount of lithium ions during charging and discharging of the battery. Hence, the design of the integrated antenna needs to account for the unknown electromagnetic parameters of the doped Pedot:PSS. To this purpose, a combined experimental/numerical identification procedure is also presented to obtain the permittivity and conductivity of the charged conductive polymer. gaetano.marrocco@uniroma2.it, caccami@ing.uniroma2.it. M. P. Hogan and M. Alfredsson are with the School of Physical Sciences, University of Kent, Canterbury, Kent, UK. email: mh618@kent.ac.uk, M.L.Alfredsson@ kent.ac.uk. J. C. Batchelor is with the School of Engineering, University of Kent, Canterbury, UK. email: J.C.Batchelor@kent.ac.uk. 


\section{$>$ REPLACE THIS LINE WITH YOUR PAPER IDENTIFICATION NUMBER (DOUBLE-CLICK HERE TO EDIT) $<$}

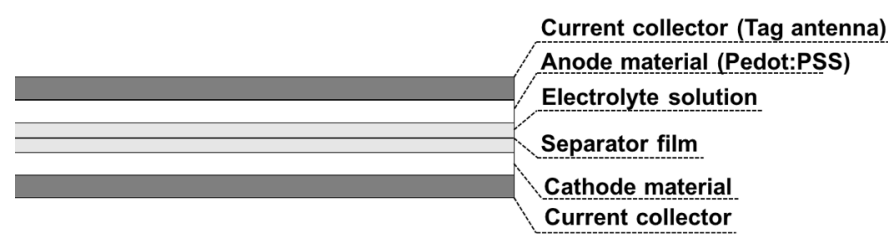

Fig. 1. A schematic cross-section of the integrated battery-antenna system.

The resulting multilayer battery-antenna system is lighter than a gram and is thinner than a millimeter making it potentially suitable for noninvasive wearable applications and wireless on-skin sensing.

The paper is organized as follows. The basics of a multilayer planar battery are summarized in Section II. The electromagnetic characterization of the doped polymer, acting as the interface between the antenna and the other battery layers, is introduced in Section III while the numerical model and the design of the integrated battery-antenna placed over a simplified human forearm phantom are shown in Section IV. Finally, Section V describes the manufacture of a fully working prototype and gives measurements of both its electrochemical properties and the communication performance when the device was attached to a volunteer's forearm.

\section{CONFIGURATION AND PRINCIPLE OF FLEXIBLE BATTERIES}

To properly describe the integrated antenna-battery, it is worth recalling the basic configuration of flexible batteries. A typical battery based on the $L i$-ion technology consists of two metallic current collectors each placed on the anode and cathode. Such current collectors allow for connection to an external circuit and guide the electrons involved in the oxidation and reduction processes that occur in the anode and cathode components. The anode (i.e., the negative electrode) usually consists of graphite intercalating lithium atoms, while the cathode (i.e., the positive electrode) is a lithium-ioncompound. Battery discharging/ charging cycles hence involve the diffusion of the lithium ions between the two electrodes through an electrolyte solution containing conducting salt and solvents. During discharging, the lithium is oxidized at the anode and is released to the electrolyte, while the electron is transferred by an external conductor to the cathode. To compensate the charges, a $\mathrm{Li}^{+}$ion migrates to the cathode. The charging process works vice versa, and $\mathrm{Li}^{+}$is reduced at the anode, while the cathode material is reoxidized. A porous separator film, moreover, is placed between the anode and cathode in order to keep the two electrodes apart to prevent electrical short circuits while also providing a means for ionic transport $[9,14]$.

This basic multilayer configuration remains unchanged for the low-profile battery that we will investigate and manufacture in this paper (Fig. 1). As described later, the negative electrode current collector will be replaced by the antenna body, while a film of conductive polymer, Pedot:PSS, i.e. (poly (3,4ethylenedioxythiophene) doped with polystyrene sulfonate [15], is used for the first time (to the best of our knowledge) as the anode material.

Pedot:PSS is a chemical interactive material (CIM), which is

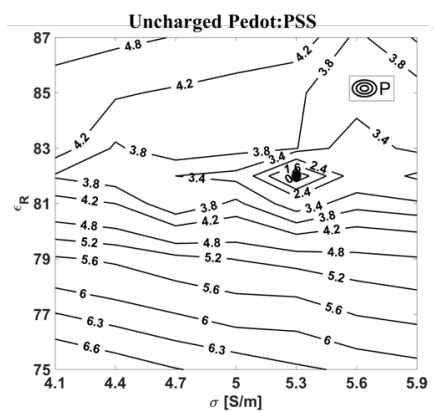

(a)

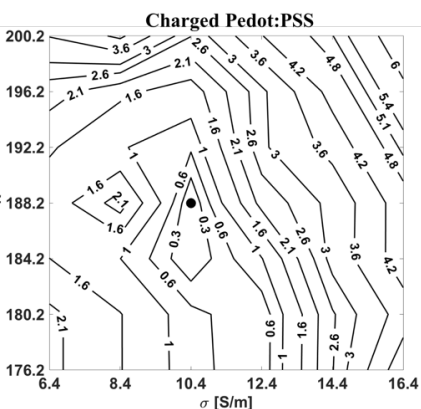

(b)
Fig. 2. Minimization of the penalty function $P$ for the identification of the electromagnetic parameters of (a) uncharged and (b) charged Pedot:PSS.

available commercially as a homogenized water based dispersion with a Pedot to PSS ratio of 1:2.5. Among conductive polymers, it possesses an excellent electrochemical stability [16, 17], is mechanically robust [18] and has been demonstrated so far to be especially attractive both as an electron conducting additive and as a binder in the battery manufacturing, providing enhanced electrochemical performance of the electrodes [19].

The Pedot:PSS layer is the direct physical interface between the antenna element and the battery parts and its electrical properties are variable over the battery lifecycle. Therefore, to ensure that the integrated antenna provides stable performance (realized gain) when the battery is both fully charged as well as during discharge, the Pedot:PSS layer requires specific electromagnetic characterization in the two extreme operative conditions (fully charged and discharged battery modes).

\section{ELECTROMAGNETIC CHARACTERIZATION OF POLYMER}

The UHF band (840-960 MHz) dielectric properties of Pedot:PSS were first characterized in [20] and [21]. The polymer was located within a doubly-folded patch antenna with a nearly uniform internal electrical field, so that it was modeled as an equivalent lumped-element. The values of the Pedot:PSS resistance and capacitance were then estimated by means of a parameter identification procedure at discrete humidity levels. However, the result was not general for other structures.

In this section, we report both an experimental UHF characterization of the dielectric properties of the Pedot:PSS when it is fully doped with conducting ions (hereafter denoted as charged Pedot:PSS) to simulate the battery charging process as well as a characterization of the pure polymer (uncharged Pedot:PSS), i.e. when the battery is out of charge.

The Pedot:PSS sample was spray coated onto the rough side of a $0.1 \mathrm{~mm}$ thick cellulose acetate (CA) sheet (area $86 \times 98$ $\mathrm{mm}^{2}$ ) which improved the polymer adherence.

In particular, for the RF characterization of the charged state polymer (when lithium ions $\mathrm{Li}^{+}$migrate from the cathode to the anode) the Pedot:PSS was doped with lithium ions $\left(\mathrm{Li}^{+}\right)$by adding lithium salt (Lithium Hexafluorophosphate, $\mathrm{LiPF}_{6}$ ) into aqueous Pedot:PSS solution (10 wt $\%$ salt to $90 \mathrm{wt} \%$ Pedot:PSS, once water was evaporated). The solution was first ultrasonicated and then spray coated over a CA sheet and left to dry in ambient conditions. 


\section{HERE TO EDIT) $<$}

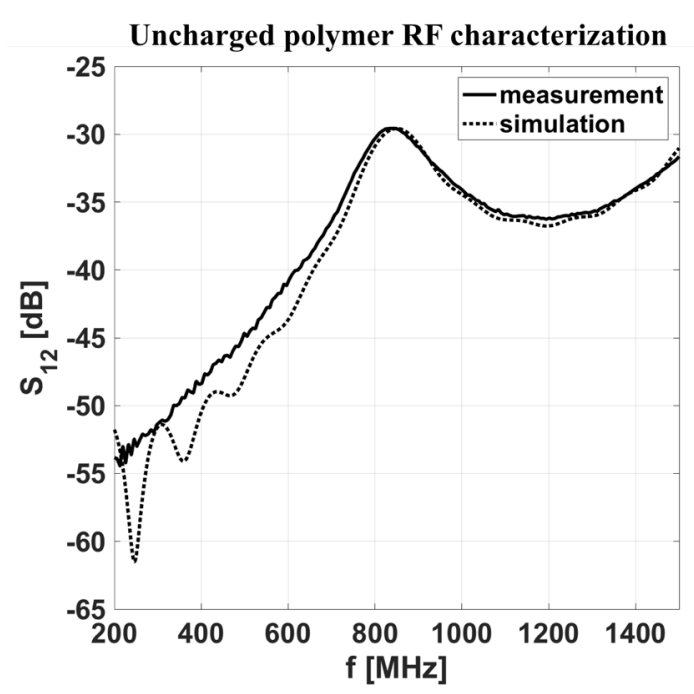

(a)

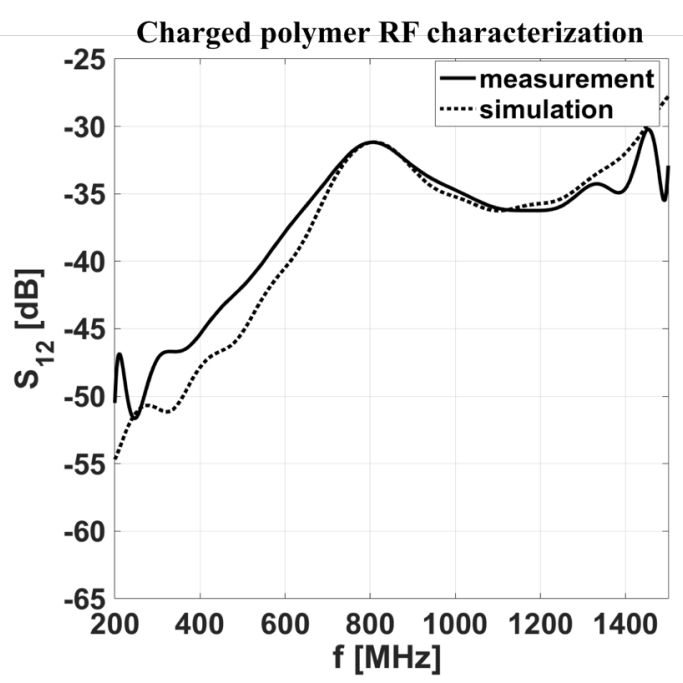

(b)

Fig. 3. Superposition of the $S_{12}$ measurements and the corresponding simulations vs. frequency (a) for the uncharged polymer RF characterization (CA sheet coated by pure Pedot:PSS) and (b) for the charged polymer RF characterization (CA sheet coated by Pedot:PSS doped with lithium ions simulating the battery charging).

The permittivity and the electrical conductivity of the two polymer films were then obtained by a numerical-experimental parameter-identification procedure. This procedure will only return a single value for the permittivity and conductivity that are hence assumed to be stable at least in the UHF RFID band, as already verified for lossy biological tissues [22]. The dielectric measurement involved a multilayer microstrip ring resonator [23] with the ring and ground plane/feed in separate planes [24]. Each sample was placed between the ring and ground layers and the $S_{12}$ was measured across the band of interest with a Vector Network Analyzer. Then, the $S_{12}$ peak frequency $f_{R}$ is detected as well the quality factor:

$$
Q=\frac{f_{R}}{\left|f_{\max }-f_{\min }\right|_{-3 d B}}
$$

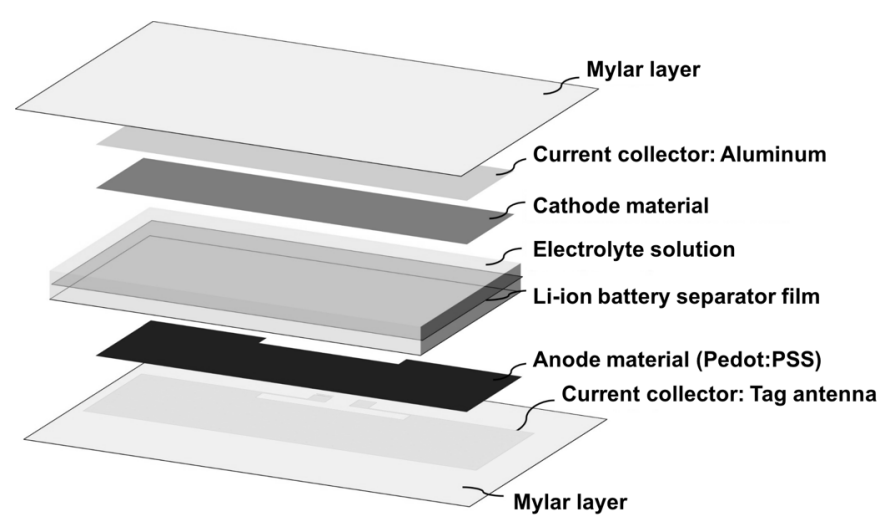

Fig. 4. An exploded view of the multilayer integrated battery-antenna system.

where $f_{\max }$ and $f_{\min }$ are respectively the upper and lower frequency half-power $S_{12}$ values. A numerical model of the ring resonator including the $\mathrm{CA}$ sheet $\left(\varepsilon_{\mathrm{R}}=2.9\right.$ and $\sigma=0.059 \mathrm{~S} / \mathrm{m}$, [25]) was created with the Pedot:PSS dielectric properties as parametric variables $\left(\varepsilon_{R}, \sigma\right)$ to be identified by minimizing the following penalty function:

$$
\begin{aligned}
P=w_{1} \mid f_{R, \text { meas }}- & f_{R, \operatorname{sim}}\left(\varepsilon_{R}, \sigma\right) \mid \\
& +w_{2}\left|Q_{\text {meas }}-Q_{\text {sim }}\left(\varepsilon_{R}, \sigma\right)\right|
\end{aligned}
$$

with the weighting coefficients $w_{1}=w_{2}=0.5$. The twolevel (coarse and fine) parameter identification comprised a preliminary manual empirical search for the selection of the appropriate range (Fig. 2) for $\left(\varepsilon_{R}, \sigma\right)$ starting from the limited available knowledge in [20], [21]. Then, the optimal solution was obtained by the application of the Trust Region Framework local optimization method [26]. The identification finally returned $\varepsilon_{\mathrm{R}}=82$ and $\sigma=5.3 \mathrm{~S} / \mathrm{m}$ for the uncharged case and $\varepsilon_{R}$ $=188.2$ and $\sigma=10.4 \mathrm{~S} / \mathrm{m}$ for the charged Pedot:PSS case. By using these values, the electromagnetic model fits accurately to the measured data $S_{12}$ (Fig. 3). It is worth noticing that the presence of $\mathrm{Li}^{+}$causes a downward shift of the $S_{12}$ peak frequency versus the uncharged Pedot:PSS that correlates with a significant increase in permittivity and a reduction of the quality factor according to an increase in the electrical conductivity.

These dielectric values for the polymeric film will be used in the numerical modeling described in Section IV to enable accurate tag antenna design for both battery discharging and charging conditions.

\section{INTEGRATION OF THE BATTERY AND SKIN UHF RFID TAG}

An exploded view of the multilayer structure of the proposed integrated battery-antenna system (IBAS) is shown in Fig. 4. The battery anode current collector is replaced by an RFID tag antenna formed of a copper sheet and coated by a thin layer of conductive Pedot:PSS. The positive electrode current collector is a layer of aluminum on which the cathode material is deposited. To protect the human skin and exclude moisture, the device is enclosed between two layers of $50 \mu \mathrm{m}$ thick Mylar 

HERE TO EDIT) $<$

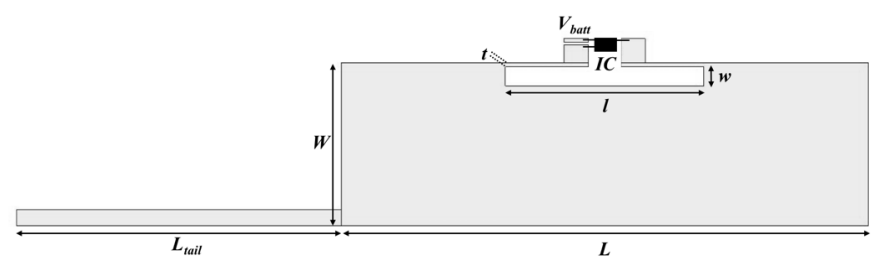

Fig. 5. Layout of the nested slotline RFID antenna. Size in $m m: L=65 ; W=$ $20 ; l=25 ; w=2.2 ; t=0.5 ; L_{\text {tail }}=40$.

polyester film $\left(\varepsilon_{\mathrm{R}}=2.8\right.$ and $\tan \delta=0.008 \mathrm{~S} / \mathrm{m}$ at $1 \mathrm{GHz}$ and $\left.25^{\circ} \mathrm{C},[27]\right)$.

\section{A. Antenna layout}

The RFID tag antenna is a nested slotline design derived from [28]. Figure 5 shows the geometry of the tag which consists of a slot at a small distance $t$ from the upper edge. Two short coplanar lines, separated by a gap, extend out of the slot center to the RFID microchip, which is connected across the two lines. An EM4325-TSSOP8 RFID IC transponder [29] was selected as it is suitable to be driven in battery assisted mode. Moreover, the chip has been widely used in epidermal electronics applications [30] as it contains an integrated temperature sensor with programmable dynamic range between $-40 /+64^{\circ} \mathrm{C}$ and a resolution of $\pm 0.25^{\circ} \mathrm{C}$. In battery-less mode the electrical parameters are: input impedance $Z_{\text {chip }}=23.3-j 145$ $\Omega$ at $866 \mathrm{MHz}$ and power sensitivity $p_{\text {chip }}=-8 \mathrm{dBm}$; while in battery-assisted (BAP) mode: $Z_{\text {chip }}=7.4-j 122 \Omega$ at $866 \mathrm{MHz}$ with several selectable $p_{\text {chip }}$ levels down to $-31 \mathrm{dBm}$.

The $V_{\text {batt }}$ pin of the EM4325 has to be connected to the battery cathode, and a copper tail $L_{\text {tail }}$ is necessary to enable the connections for charging the battery. The outer sizes of the antenna are set to $L=65 \mathrm{~mm}$ and $W=20 \mathrm{~mm}$ as a trade-off between a reasonable antenna gain and a moderate overall antenna size.

The response of the antenna was simulated with the Finite Difference Time Domain (FDTD) solver of CST Microwave Studio 2017 [31]. The Pedot:PSS was modelled as a $35 \mu \mathrm{m}$ thick homogeneous brick with dielectric properties for the uncharged and charged states that were obtained in Section III. For the sake of simplicity and the simulation speed, the model of the device also included a simplified model phantom of the human forearm consisting of a finite-size-layered block (see the inset in Fig. 8). The thickness of the different layers and the frequency-dependent average electromagnetic parameters, resembling the human body tissues [22], [32] - [34], are shown in Table I.

TABLE I

PHYSICAL AND GEOMETRICAL PARAMETERS OF THE LAYERED ANATOMICAL MODEL AT $870 \mathrm{MHZ}$

\begin{tabular}{llll}
\hline \hline Layer & $\varepsilon_{\mathrm{R}}$ & $\sigma[\mathrm{S} / \mathrm{m}]$ & Thickness $[\mathrm{mm}]$ \\
\hline Skin + Fat & 14.5 & 0.25 & 3 \\
Muscle & 55.1 & 0.93 & 15 \\
Bone cancellous & 20.8 & 0.33 & 5
\end{tabular}

The electromagnetic performance of the IBAS was referred to the power transmission coefficient:

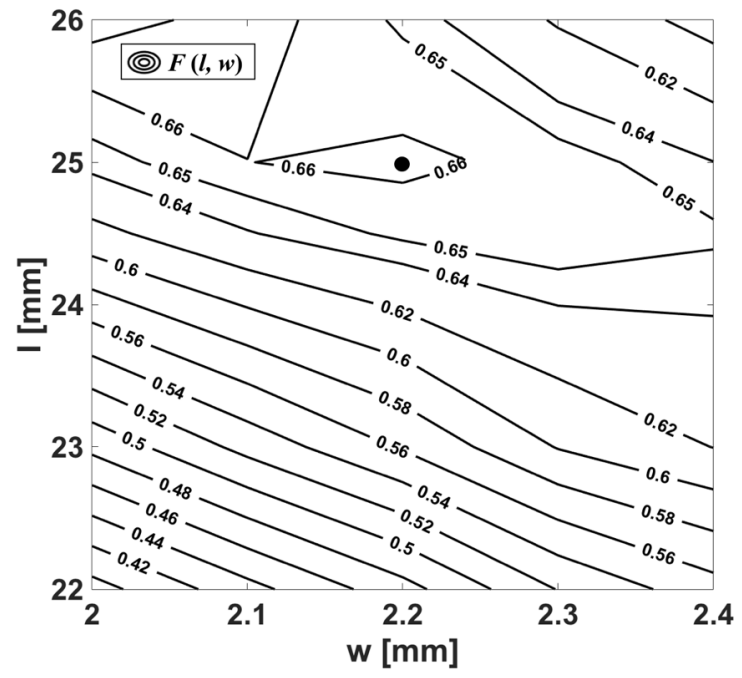

Fig. 6. Map of the fitness function $F(l, w)$ in Eq. (4) calculated by numerical simulations for the antenna-microchip impedance matching at $870 \mathrm{MHz}$.

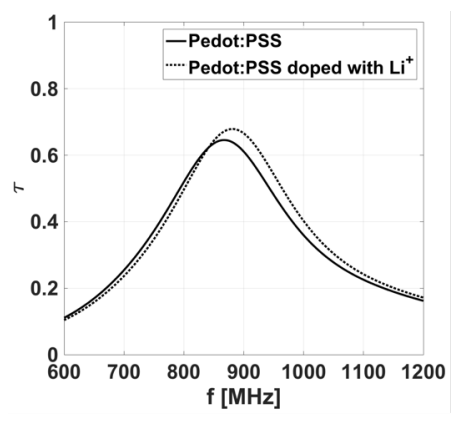

(a)

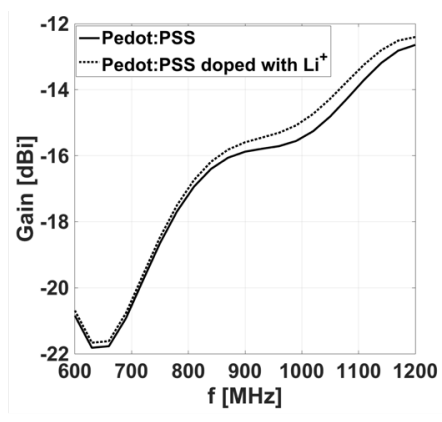

(b)
Fig. 7. Simulated (a) power transmission coefficient $\tau$ and (b) gain vs. frequency of the integrated UHF RFID battery-antenna system placed on the layered human body phantom for Pedot:PSS pure and doped with lithium salt to simulate the battery cycling process.

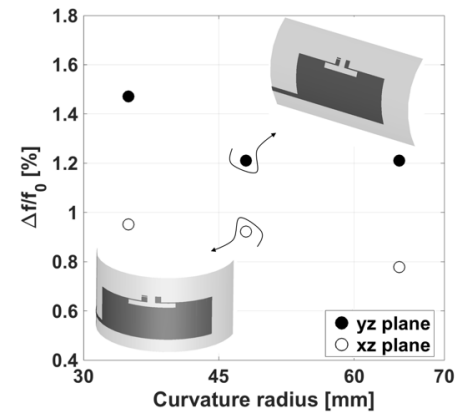

(a)

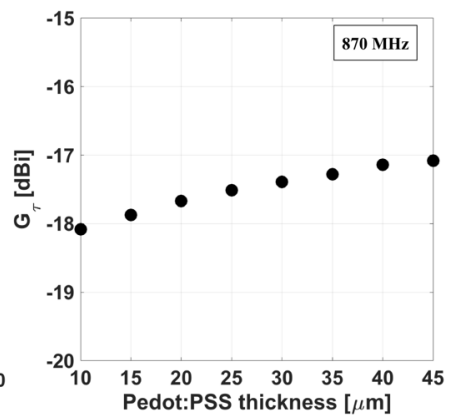

(b)
Fig. 8. (a) Relative variation of the frequency $f$ with respect to that of flat configuration $f_{0}$ when the IBAS is bent along the two principal planes, $\mathrm{xz}$ and $\mathrm{yz}$, with different curvature radii $r=\{35,48,65\} \mathrm{mm}$. (b) Simulated realized gain of IBAS at $870 \mathrm{MHz}$ when the thickness of the conductive polymer is increased from $10 \mu \mathrm{m}$ up to $45 \mu \mathrm{m}$. 


\section{$>$ REPLACE THIS LINE WITH YOUR PAPER IDENTIFICATION NUMBER (DOUBLE-CLICK HERE TO EDIT) <}

$$
\tau=\frac{4 R_{A} R_{\text {chip }}}{\left|Z_{A}+Z_{\text {chip }}\right|^{2}} \leq 1
$$

which accounts for the impedance mismatch between the antenna and the microchip. The best trade-off between the $\tau$ values for the discharging/charging states of the battery was established through the fitness function to be maximized with respect to the antenna's slot sizes $\{l, w\}$ :

$$
F(l, w)=\frac{1}{2}\left(\tau_{\text {uncharged }}+\tau_{\text {charged }}\right)
$$

for the EM4325 BAP mode input impedance, where $\tau_{\text {uncharged }}$ and $\tau_{\text {charged }}$ refer respectively to the discharged and charged Pedot:PSS layer. The map of the fitness function in Fig. 6 indicates the optimal couplet $l=25 \mathrm{~mm}$ and $w=2.2 \mathrm{~mm}$ and the corresponding power transmission coefficient curves for the uncharged and charged Pedot:PSS states (Fig. 7(a)) shows a $15 \mathrm{MHz}$ frequency shift between the charged and uncharged situations with a negligible change in the peak level. The bandwidth for $\tau>0.5$ is $162 \mathrm{MHz}(18 \%)$. The simulated broadside antenna gain in Fig. 7(b) indicates the antenna radiation efficiency is not sensitive to the polymer status with only $0.3 \mathrm{dBi}$ difference between the uncharged and charged curves at $870 \mathrm{MHz}$. The antenna radiation gain is of the order of $-16 \mathrm{dBi}$ around the frequency of $870 \mathrm{MHz}$ where the antenna have been optimized. This value is comparable with that of other reported epidermal antennas which span between -20 and $-10 \mathrm{dBi}[35]$ and is clearly expected because of the close interaction between the antenna and the human tissues with a resulting significant power loss.

The sensitivity of the IBAS to moderate bending emulating the natural curvature of the human body region is reported in Fig. 8(a). The antenna (together with the multilayer model) was bent along the two principal $x z$ and $y z$ planes, according to typical curvature radii $r=\{35,48,65\} \mathrm{mm}$ of the female and male human arm. The frequency shift of the power transfer coefficient peak with respect to the unbent IBAS was hence numerically evaluated. The resulting change of approximately

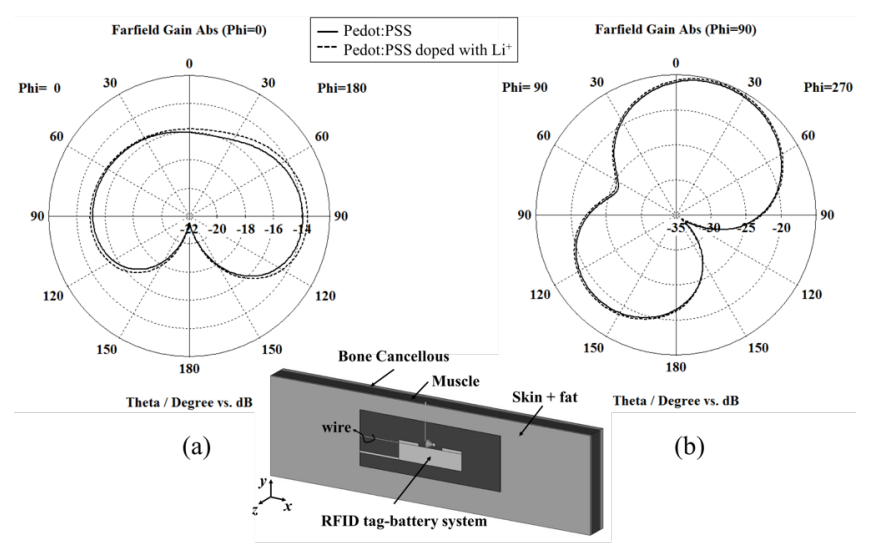

Fig. 9. Polar plots of simulated gain of the antenna-battery system in (a) $y-z$ plane and in (b) $x-z$ plane when it is mounted on reference model of the forearm. The inset shows the UHF RFID tag-battery system attached onto a phantom of a human forearm.

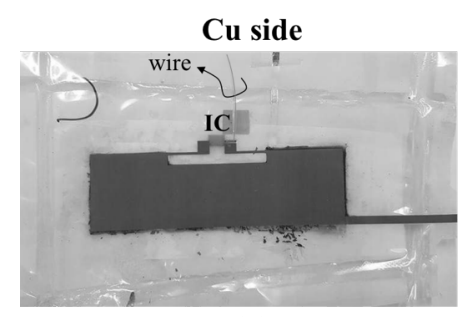

(a)

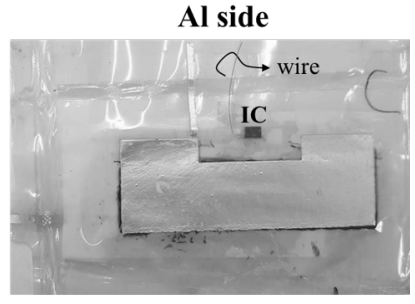

(b)
Fig. 10. Prototype of the tag antenna integrated into the multilayer structure of the polymer-based battery: (a) top view; (b) rear view.

$2 \%$ will not degrade the power transfer coefficient below $\tau=$ 0.5 owing to the large bandwidth of the antenna. Moreover, as the gain is practically constant over an even larger band (Fig. 7(b)), the expected degradation of the read range will be at most $12 \%$ the maximum read distance of the unbent IBAS.

Fig. 8(b) shows a parametric analysis of the antenna realized gain at $870 \mathrm{MHz}$, obtained by FDTD simulations, with respect to the Pedot:PSS thickness in the range $10 \mu \mathrm{m}$ to $45 \mu \mathrm{m}$. As the thickness increases, the IBAS realized gain tends to an approximately constant value with $1 \mathrm{dBi}$ overall variation in the whole considered range. In particular, for values of thickness greater than $25 \mu \mathrm{m}$, the variation of the realized gain is $0.5 \mathrm{dBi}$ compared to the maximum value.

Finally, the simulated gain polar plots in both $x-z$ and $y-z$ cutplanes of the antenna are depicted in Fig. 9. While the presence of the phantom has an expected significant effect on the radiation, the charge status of the battery has very little impact on the directivity.

\section{PROTOTYPE AND MEASUREMENTS}

\section{A. Prototype}

A prototype of the whole integrated battery-epidermal tag antenna system (Fig. 10) was fabricated using conventional wet etching of a copper clad Mylar sheet for the antenna $(60 \mu \mathrm{m}$ total thickness). A $0.2 \mathrm{~mm}$ diameter wire was soldered to connect the chip $V_{\text {batt }}$ pin to the battery positive terminal (the aluminum layer coated by the cathode material). The cathode material was a mixture of $\mathrm{LiNi}_{0.5} \mathrm{Mn}_{1.5} \mathrm{O}_{4}(80 \%$ wt), Super $\mathrm{P}$ carbon black $(10 \% \mathrm{wt})$ and $\mathrm{PVdF}(10 \% \mathrm{wt}$, (Poly(vinylidene fluoride) average Mw-534,000 by GPC, powder, Sigma Aldrich) and deposited by Doctor blade printing on the $15 \mu \mathrm{m}$ thick aluminum layer.

The anode was formed of $10 \mu 1$ of Pedot:PSS solution, which was ultrasonicated for 10 minutes and deposited by spray coating on the antenna copper.

A screw gauge (Hilka brand micrometer) with a resolution of $10 \mu \mathrm{m}$ was used for measuring the layers of the Pedot:PSS polymer and the cathode material after their deposition on copper and aluminum foils, respectively.

As the battery materials are air-sensitive, the different parts were assembled inside a glovebox filled with Argon gas. The electrolytic solution, which is a mixture of a conducting salt and solvent, i.e. Lithium hexafluorophosphate solution in ethylene carbonate and diethyl carbonate $\left(1 \mathrm{M} \mathrm{LiPF}_{6}\right.$ in $\mathrm{EC} / \mathrm{DEC}=50 / 50$ $(\mathrm{v} / \mathrm{v})$, Sigma Aldrich), was dispensed by a syringe. A 


\section{$>$ REPLACE THIS LINE WITH YOUR PAPER IDENTIFICATION NUMBER (DOUBLE-CLICK HERE TO EDIT) $<$}

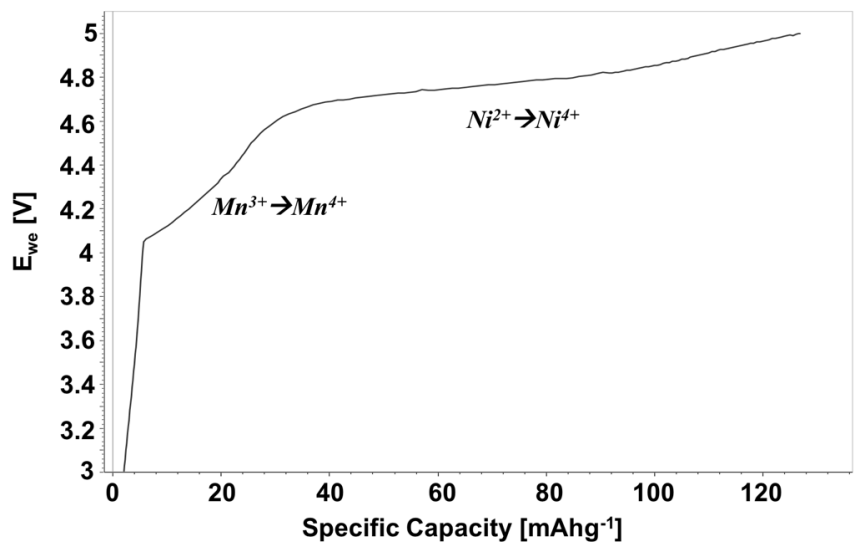

Fig. 11. Voltage - capacity curves for flat plateau corresponding to the operating potential $4.7 \mathrm{~V}$ vs. $\mathrm{Li} / \mathrm{Li}^{+}$.

microporous trilayer membrane (Celgard 2325 membrane, $25 \mu \mathrm{m}$ thick and $85 \mathrm{~mm}$ wide), mainly consisting of polypropylene PP and polyethylene PE, was cut to a prescribed length and used as the $L i$-ion battery separator film [36].

The components were stacked and a small amount of electrolyte was injected to saturate the anode coating on the copper antenna. The separator film was placed on the anode material (the Pedot:PSS film) and a small amount of electrolyte was injected on the separator film to soak the cathode material. The cathode material film, deposited on the aluminum layer, was then placed in contact with the separator membrane. Finally, a thin Mylar polyester film was placed in contact with the aluminum substrate and the composite battery-antenna system was compressed and vacuum sealed.

\section{B. Battery Capacity}

The polymeric battery capacity and charging voltage were tested [14]. The charge voltage cycling curve is shown in Fig. 11 and exhibits two well-defined plateaus: the first at 4.1 $\mathrm{V}$, corresponding to the oxidation state of $\mathrm{Mn}^{3+}$ to $\mathrm{Mn}^{4+}$, and a second plateau at $4.7 \mathrm{~V}$ corresponding to the oxidation state of $\mathrm{Ni}^{2+}$ to $\mathrm{Ni}^{4+}$. These values compare well with published data on $\mathrm{LiNi}_{0.5} \mathrm{Mn}_{1.5} \mathrm{O}_{4}$ half cells [37], [38]. The corresponding specific charge capacity of the battery, $C_{\text {spec }}$ was $125 \mathrm{mAhg}^{-1}$.

\section{Realized gain}

The measured communication performance of the integrated system was characterized through the realized gain defined as $G_{\tau}=G \cdot \tau$, i.e. the radiation gain of the tag scaled by the power transfer to the IC. The interrogating measurement set-up comprised a Voyantic Tagformance UHF RFID measurement system [39] connected to a linearly-polarized patch antenna with gain $G_{R}=8.15 d B i$ placed at a fixed distance $d=30 \mathrm{~cm}$ from the tag. The system measured the turn-on power $P_{\text {in }}^{\text {to }}$ [40], [41], which corresponds to the minimum input power $P_{\text {in }}$ feeding the reader antenna in order to activate the tag chip. The realized gain was hence obtained from the turn-on power measurement by inverting the Friis formula:

$$
G_{\tau}=G \cdot \tau=\left(\frac{4 \pi d}{\lambda}\right)^{2} \frac{p_{c h i p}}{G_{R} P_{i n}^{t o} \eta_{P}}
$$

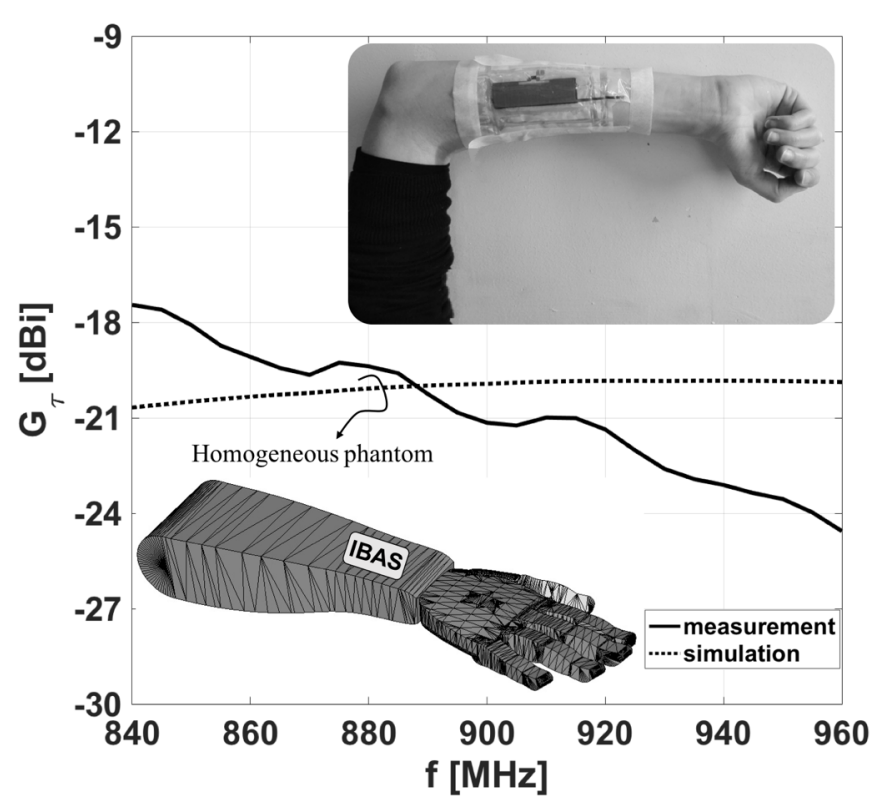

Fig. 12. Comparison between the measured realized gain along the antenna axis (broadside observation) and the simulated data having considered a homogeneous $\left(\varepsilon_{\mathrm{R}}=30\right.$ and $\left.\sigma=0.62 \mathrm{~S} / \mathrm{m}\right)$ arm phantom [42] as reference. The inset shows the prototype mounted onto a volunteer's forearm.

where $\eta_{P}$ is the polarization efficiency between the reader and the tag and $p_{\text {chip }}$ is the power sensitivity of the microchip.

Fig. 12 shows the realized gain which was measured after charging the battery and when the tag was placed directly onto a volunteer's forearm. Experimental data were compared with simulations involving a more realistic homogeneous $\left(\varepsilon_{\mathrm{R}}=30\right.$ and $\sigma=0.62 \mathrm{~S} / \mathrm{m}$ ) arm phantom borrowed from [42]. Despite of a mutual frequency shift between the gain responses, probably due to uncertainty of the constitutive parameters of the real arm

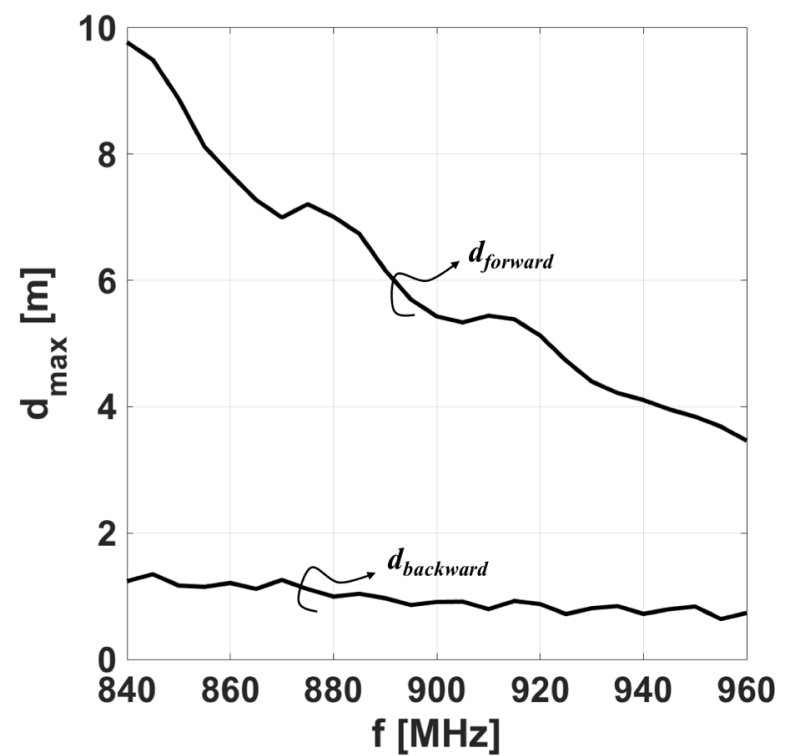

Fig. 13. Theoretical maximum read distances for $3.2 \mathrm{~W}$ EIRP interrogating power, extrapolated from measurements carried out by means of the Tagformance reader for both direct and reverse RFID links. 

HERE TO EDIT) $<$

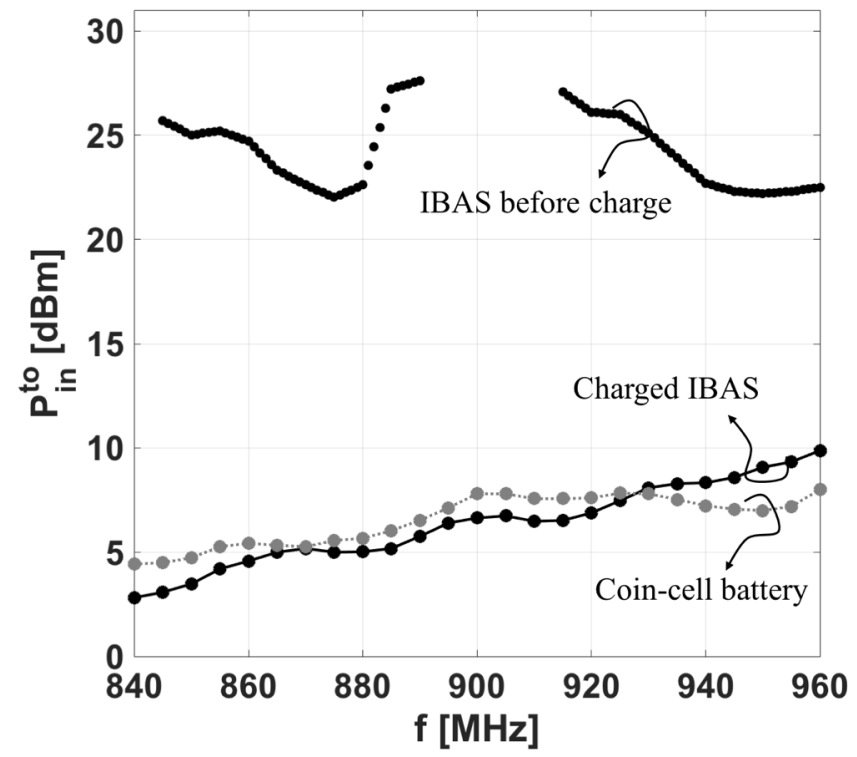

Fig. 14. Comparison among the measured turn-on powers vs. frequency of the integrated battery-antenna system (IBAS), before and after charge, and of the conventional coin cell battery.

and the unknown frequency variation of the microchip impedance that was here neglected, the overall tag behavior can be reproduced by the numerical model with a maximum error of $3 \mathrm{dBi}$ in the whole RFID band.

\section{Read distance}

Owing to the high sensitivity of the chip, the estimation of the maximum read distance requires to evaluate both the forward and backward links. The forward maximum read distance is derived from (5) by assuming $G_{R} P_{\text {in }}=3.2 \mathrm{WEIRP}$ (the maximum allowed by European regulations), while the backward maximum read distance is derived from the radar equation [43] as:

$$
d_{\text {backward }}=\left(\frac{\lambda}{4 \pi}\right) \sqrt[4]{\frac{P_{\text {in }} G_{R}^{2} G_{\text {tag }}^{2} \eta_{P}^{2} \rho}{p_{\text {reader }}}}
$$

where $\rho$ is the tag backscatter modulation loss. By assuming the chip sensitivity in BAP mode to be $p_{\text {chip }}=-28 \mathrm{dBm}$, and the reader sensitivity to be $p_{\text {reader }}=-75 \mathrm{dBm}$, the theoretical maximum read distances are equal to $d_{\text {forward }}=6.9 \mathrm{~m}$ and $d_{\text {backward }}=1.3 \mathrm{~m}$ at $870 \mathrm{MHz}$ (Fig. 13). Therefore, the communication bottleneck is the backward link which can occur in the case of battery assisted tags where the reader sensitivity requires improvement [43, 44]. As it stands, the proposed integrated device is well suited for a wide range of applications in healthcare and security. In particular, the computed reading distance of $1.3-3 \mathrm{~m}$, depending on the reader and the surrounding environment, is sufficient to enable data downloading from the device working as an epidermal datalogger. Some examples involve the manual or/and automatic reading for data collection when a user passes an interrogating base station, such as a door gate [30] or a handheld reader.

Finally, the RFID BAP performance of the prototype composite battery-antenna system was compared to that of the same tag connected to a standard $1.5 \mathrm{~V}$ voltage coin cell battery (Eunicell AG13 alkaline button cell with $11.6 \mathrm{~mm}$ diameter by $5.4 \mathrm{~mm}$ height, [45]). The measured profiles of the turn-on powers versus frequency are shown in Fig. 14 and are comparable. Figure 14 also shows the measurement of the turnon power before charging the integrated battery-antenna system. By comparing the power values before and after the integrated battery-antenna system is charged, a difference of approximately $20 \mathrm{dBm}$ in the turn-on power is observed. This, as expected, is related to the sensitivity modes of the microchip, which switches from $-8 \mathrm{dBm}$ in passive mode to $-28 \mathrm{dBm}$ in the BAP condition.

\section{E. Specific Absorption Rate}

It is worth analyzing the localized specific absorption rate (SAR) averaged over $10 \mathrm{~g}$ of tissue. SAR is required to be smaller than $4 \mathrm{~W} / \mathrm{kg}$ for the exposure of limbs and less than 2 $\mathrm{W} / \mathrm{kg}$ for head and trunks [46] averaged over $10 \mathrm{~g}$ of tissues and time-averaged over 6 minutes of exposure. The SAR distribution in the phantom of the human arm, computed when IBAS is stuck on skin and by assuming the available power at the reader's antenna equal to $\mathrm{P}_{\mathrm{in}}=1 \mathrm{~W}$, is reported in Fig. 15 . The estimated SAR value $(0.05 \mathrm{~W} / \mathrm{kg})$ is below the limits imposed by regulations, so that the UHF interrogation of the integrated system can be considered completely compliant with SAR exposure limits.
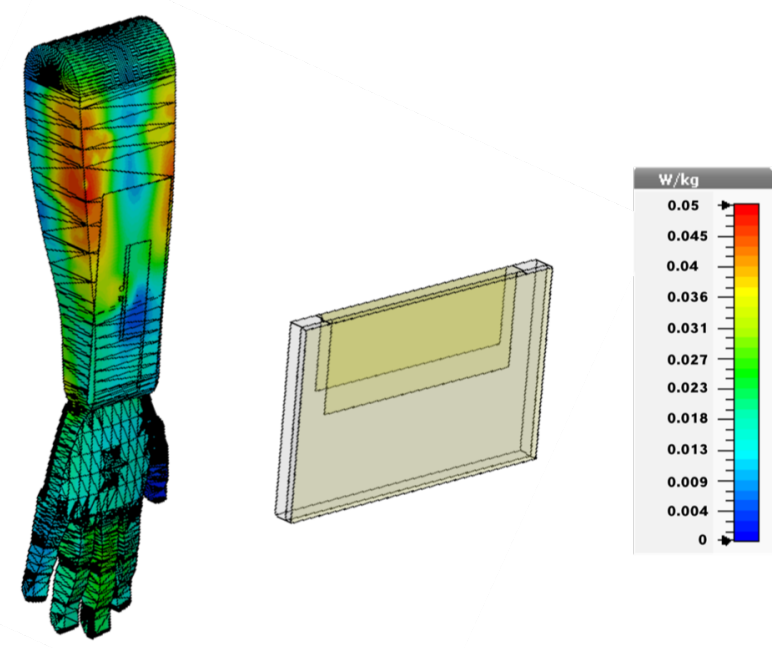

Fig. 15. SAR distribution produced in the arm by the reader's antenna placed at $30 \mathrm{~cm}$ from the IBAS and fed by $1 \mathrm{~W}$ input power.

\section{DISCUSSION AND CONCLUSION}

We have presented the first tightly integrated battery-assisted tag, as a compact device, suitable for direct mounting on the skin. The device is composed of an epidermal RFID antenna embedded as the current collector at the anode of a low-profile flexible battery made of conductive polymer. The resulting antenna is ultra-thin (approximately $200 \mu \mathrm{m}$ ) for noninvasive wearable applications and can be used as an epidermal datalogger. 


\section{$>$ REPLACE THIS LINE WITH YOUR PAPER IDENTIFICATION NUMBER (DOUBLE-CLICK HERE TO EDIT) $<$}

Microwave characterization of the Pedot:PSS film used here for the first time as the anode material and acting as antenna/battery interface, has enabled modeling of the integrated tag in the charged and uncharged states. Moreover, we demonstrated that the communication performances of the prototype approaches that of a conventional coin cell battery having comparable voltage value, when connected to the device.

In this first prototype, protection from electrolyte contact with skin is necessary to prevent skin irritations and burns. This has been done by laminating the IBAS between two Mylar polyester films to prevent leaking from the battery. For further product development a better solution will involve a solid polymer electrolyte (SPE), such as a poly(ethylene glycol) diacrylate (pegda) based electrolyte [47]. This coating will not only improve safety by preventing leaking, but also reduce the flammability risk of the IBAS and additionally enable single production line manufacturing.

Some critical issues still require additional investigations and improvements, especially concerning the reliability of the device. Therefore, effort must be devoted to better sealing of the different battery components, as well as the adoption of a standard procedure of deposition of the polymer material to obtain a more uniform chemical coating. The prototype battery life was approximately 1 hour, though this could be improved by introducing appropriate DC current limiting circuitry.

Finally, although the resulting device is mechanically flexible for a conformal placement onto the human body with electromagnetic performance relatively unaffected by a moderate curvature, the impact on the electrochemical properties of the battery is currently unknown and will be the topic of a further paper.

\section{REFERENCES}

[1] J. Rogers, R. Ghaffari, D. H. Kim, "Stretchable Bioelectronics for Medical Devices and Systems," in Microsystems and Nanosystems, Davos, Switzerland:Springer, 2016.

[2] S. Choi, H. Lee, R. Ghaffari, T. Hyeon, and D. Kim, "Recent Advances in Flexible and Stretchable Bio-Electronic Devices Integrated with Nanomaterials," Adv. Mater., vol. 28, no. 22, pp. 4203 - 4218, Jun. 2016.

[3] K. K. Fu, J. Cheng, T. Li, and L. Hu, "Flexible Batteries: From Mechanics to Devices," ACS Energy Let., , vol. 1, no. 5, pp. 1065 - 1079, Nov. 2016.

[4] A. J. Bandodkar, "Review - Wearable Biofuel Cells: Past, Present and Future," J. Electrochem. Soc., vol. 164, no. 3, pp. H3007-H3014, 2017.

[5] J. Kim, R. Kumar, A. J. Bandodkar, and J. Wang, "Advanced Materials for Printed Wearable Electrochemical Devices: A Review," $A d v$. Electron. Mater., vol. 3, no. 1, 1600260, pp. 1 - 15, Jan. 2017.

[6] S. Xu, Y. Zhang, J. Cho, J. Lee, X. Huang, L. Jia, J. A. Fan, Y. Su, J. Su, H. Zhang, H. Cheng, B. Lu, C. Yu, C. Chuang, T. Kim, T. Song, K. Shigeta, S. Kang, C. Dagdeviren, I. Petrov, P. V. Braun, Y. Huang, U. Paik, and J. A. Rogers, "Stretchable batteries with self-similar serpentine interconnects and integrated wireless recharging systems, Nat. Commun. 4, no. 1543, pp. 1 - 8, Feb. 2013.

[7] S. Berchmans, A. J. Bandodkar, W. Jia, J. Ramirez, Y. S. Meng and J. Wang, "An epidermal alkaline rechargeable $\mathrm{Ag}-\mathrm{Zn}$ printable tattoo battery for wearable electronics," $J$ Mater Chem A, vol. 2, no. 38, pp. 15788 - 15795, Jul. 2014.

[8] Nagatomo, C. Ichikawa, and O. Omoto, "All-plastic batteries with polyacetylene electrodes," J. Electrochem. Soc., vol. 134, no. 2, pp. 305 308, Feb. 1987

[9] S. Muench, A. Wild, C. Friebe, B. Häpler, T. Janoschka, and U. S. Schubert, "Polymer-Based Organic Batteries," Chem. Rev., vol. 116, no. 16, pp. 9438 - 9484, Aug. 2016.
[10] C. T. Rodenbeck, "Planar Miniature RFID Antennas Suitable for Integration With Batteries," IEEE Trans. Antennas Propag., vol. 54, no. 12, pp. 3700-3706, Dec. 2006.

[11] J. Yeo, S. G. Moon, and J. Y. Jung, "Antennas for a battery-assisted RFID tag with thin and flexible film batteries," Microw Opt Techn Let, vol. 50, no. 2, pp. 494 - 498, Feb. 2008.

[12] M. Woods and J. C. Batchelor, "Low-profile Slot Antenna Integrated with a Thin Polymer Non-Metallic Battery," in Proc. 2013 Loughborough Ant. Propag. Conf. (LAPC), Loughborough, 2013, pp. 441 - 445.

[13] M. A. Woods, O. O. Rakibet, P. R. Young, R. Luck, M. L. Alfredsson, and J. C. Batchelor, "Integrated antenna-battery for low-profile short range communications," in Proc. 8th Eur. Conf. Ant. Propag. (EuCAP 2014), The Hague, 2014, pp. 1754-1756.

[14] D. Linden, T. B. Reddy, "Handbook of Batteries," 3rd ed., New York: McGraw-Hill, 2002.

[15] A. Pasha, A. S. Roy, M. V. Murugendrappa, O. A. Al-Hartomy, S. Khasim, "Conductivity and dielectric properties of PEDOT-PSS doped DMSO nano composite thin films," J Mater Sci Mater El, vol. 27, no. 8, pp. 8332-8339, Aug. 2016.

[16] F. M. Courtel, S. Niketic, D. Duguay, Y. Abu-Lebdeh, I. J. Davidson, "Water-Soluble Binders for MCMB Carbon Anodes for Lithium-Ion Batteries," J Power Sources, vol. 196, no. 4, pp. 2128 - 2134, Feb. 2011.

[17] J. Roncali, P. Blanchard, and P. Frere, "3,4-Ethylenedioxythiophene (EDOT) as a Versatile Building Block for Advanced Functional ПConjugated Systems," J Mater Chem, vol. 15, no. 16, pp. 1589 - 1610, 2005.

[18] D. J. Lipomi, J. A. Lee, M. Vosgueritchian, B. C. K. Tee, J. A. Bolander; Z. Bao, "Electronic Properties of Transparent Conductive Films of Pedot:Pss on Stretchable Substrates," Chem. Mater., vol. 24, no. 2, pp. 373 - 382, 2012.

[19] T. M. Higgins, S.-H. Park, P. J. King, C. J. Zhang, N. McEvoy, N. C. Berner, D. Daly, A. Shmeliov, U.Khan, G. Duesberg, V. Nicolosi, and J. N. Coleman, "A Commercial Conducting Polymer as Both Binder and Conductive Additive for Silicon Nanoparticle-Based Lithium-Ion Battery Negative Electrodes," ACS Nano, vol. 10, no. 3, pp. 3702 - 3713, 2016.

[20] S. Manzari, C. Occhiuzzi, S. Nawale, A. Catini, C. Di Natale, and G. Marrocco, "Humidity Sensing by Polymer-loaded UHF RFID Antennas," IEEE Sens. J., vol. 12, no. 9, pp. 2851-2858, Sep. 2012.

[21] S. Manzari and G. Marrocco, "Modeling and Applications of a ChemicalLoaded UHF RFID Sensing Antenna With Tuning Capability," IEEE Trans. Antennas Propag., vol. 62, no. 1, pp. 94-101, Jan. 2014.

[22] C. Gabriel and S. Gabriel, (1996), "Compilation of the Dielectric Properties of Body Tissues at RF and Microwave Frequencies". [Online]. Available: http://niremf.ifac.cnr.it/docs/DIELECTRIC/home.html.

[23] S. Amendola, C. Occhiuzzi, A. Ajovalasit, M. A. Sabatino, C. Dispenza and G. Marrocco, "Dielectric characterization of biocompatible hydrogels for application to Epidermal RFID devices," in Proc. 2015 Eur. Microw. Conf. (EuMC), Paris, 2015, pp. 379 - 382.

[24] I. Waldron, S.N. Makarov, S. Biederman, R. Ludwig, "Suspended Ring Resonator for Dielectric Constant Measurement of Foams," IEEE Microw Wirel Compon Lett., IEEE, vol. 16, no. 9, pp. 496 - 498, Sep. 2006.

[25] M. Schwartz, "Encyclopedia and Handbook of Materials, Parts and Finishes," CRC Press Taylor and Francis Group, 3rd Ed., 2016, pp. 1060.

[26] Ya-Xiang Yuan, "A review of trust region algorithms for optimization." [Online].Available:http://citeseerx.ist.psu.edu/viewdoc/download;jsessio nid=0244F68D26DD697EE768FCCE2C8742FD?doi=10.1.1.45.9964\&r ep=rep $1 \&$ type $=$ pdf.

[27] Mylar $^{\circledR}$ polyester film datasheet. [Online]. Available: http://usa.dupontteijinfilms.com/informationcenter/downloads/Electrical Properties.pdf.

[28] M. A. Ziai and J. C. Batchelor, "Temporary On-Skin Passive UHF RFID Transfer Tag," IEEE Trans. Antennas Propag., vol. 59, no. 10, pp. 35653571, Oct. 2011.

[29] EM4325 IC datasheet. [Online]. Available: http://www.emmicroelectronic.com.

[30] S. Amendola, G. Bovesecchi, A. Palombi, P. Coppa, and G. Marrocco, "Design, Calibration and Experimentation of an Epidermal RFID Sensor for Remote temperature Monitoring," IEEE Sens. J., vol. 16, no. 19, pp. 7250 - 7257, Oct. 2016. 


\section{$>$ REPLACE THIS LINE WITH YOUR PAPER IDENTIFICATION NUMBER (DOUBLE-CLICK HERE TO EDIT) $<$}

[31] CST Microwave Studio Suite ${ }^{\circledR}, 2017$. [Online]. Available: https://www.cst.com.

[32] J. Lorenzo, A. Lazaro, D. Girbau, R. Villarino, and E. Gil, "Analysis of On-Body Transponders Based on Frequency Selective Surfaces," Prog Electromagn Res, vol. 157, pp. 133-143, Dec. 2016.

[33] C. Gabriel, S. Gabriel, and E. Corthout, "The dielectric properties of biological tissues: I. Literature survey,” Phys Med Biol, vol. 41, no. 11, pp. 2231 - 2249, Dec. 1996.

[34] S. Gabriel, R. W. Lau, and C. Gabriel, "The dielectric properties of biological tissues: II. Measurements in the frequency range $10 \mathrm{~Hz}$ to 20 GHz," Phys Med Biol, vol. 41, no. 11, pp. 2251 - 2269, Dec. 1996.

[35] S. Amendola and G. Marrocco, "Optimal Performance of Epidermal Antennas for UHF Radiofrequency Identification and Sensing," IEEE Trans. Antennas Propag., vol. 65, no. 2, pp. 473 - 481, Feb. 2017.

[36] Celgard, "Innovative Battery Separator Technology". [Online]. Available: https://www.celgard.com/battery-innovation/.

[37] N. Amdouni, K. Zaghib, F. Gendron, A. Mauger, and C. M. Julien, "Structure and insertion properties of disordered and ordered LiNi0.5Mn1.5O4 spinels prepared by wet chemistry," Ionics, vol. 12, no. 2, pp. 117 - 126, Jul. 2006.

[38] J. Yang, X. Han, X. Zhang, F. Cheng, and J. Chen, "Spinel $\mathrm{LiNi}_{0.5} \mathrm{Mn}_{1.5} \mathrm{O}_{4}$ cathode for rechargeable lithium ion batteries: Nano vs micro, ordered phase $\left(\mathrm{P}_{3} 32\right)$ vs disordered phase (Fd3m)," Nano Res, vol. 6, no. 9, pp. 679 - 687, Sep. 2013.

[39] Tagformance lite, Voyantic Ltd. Kutojantie 11, FI-02630 Espoo, Finland. [Online]. Available: https://voyantic.com/products/tagformance-lite.

[40] P. V. Nikitin and K. V. S. Rao, "Theory and measurement of backscattering from RFID tags," IEEE Antennas Propag. Mag., vol. 48, no. 6 , pp. 212 - 218, Dec. 2006.

[41] G. Marrocco and F. Amato, "Self-sensing Passive RFID: From Theory to Tag Design and Experimentation," 2009 Eur. Microw. Conf. (EuMC), Rome, 2009, pp. 001 - 004.

[42] V. Di Cecco, S. Amendola, P. P. Valentini, and G. Marrocco, "FingerAugmented RFID System to Restore Peripherical Thermal Feeling," in Proc. 2017 IEEE Int. Conf. RFID (RFID), Phoenix, Az, 2017, pp. 54 - 60.

[43] L. W. Mayer and A. L. Scholtz, "Sensitivity and impedance measurements on UHF RFID transponder chips," in Proc. of the 2nd Int. EURASIP workshop on RFID tech., Budapest. Citeseer, 2008, pp. 001 010.

[44] S. Amendola, "Wireless and Battery-less Biointegrated Sensors for Bodycentric Internet of Things," Ph.D. dissertation, Dept. of Civil Eng. and Comp. Sci. Eng., Rome Tor Vergata Univ., Rome, IT, 2017.

[45] Eunicell battery. [Online]. Available: http://www.eunicell.com/en/web/culturenewsInfo.asp?id=54

[46] Limitation of Exposure of the General Public to Electromagnetic Fields, European Council Recommendation 1999/519/EC, Jul. 12, 1999 [Online]. Available: http://ec.europa.eu/enterprise/electr_equipment/lv/rec519.pdf, (0 Hz to $300 \mathrm{GHz}$, Official Journal, L199, 30.7.1999, p. 59).

[47] R. He, M. Echeverri, D. Ward, Y. Zhu, Thein Kyu, "Highly conductive colvent-free polymer electrolyte membrane for lithium-ion batteries: Effect of prepolymer molecular weight," J Membr Sci Technol., vol. 498, pp. 208 - 217, Jan. 2016. 